\title{
HIV-1 Infection Increases the Expression of Human Endogenous Retroviruses Type K (HERV-K) in Vitro
}

\author{
RAFAEL CONTRERAS-GALINDO, PABLO LÓPEZ, ROSA VÉLEZ, and YASUHIRO YAMAMURA
}

\begin{abstract}
Antibodies to HERV-K antigens have been linked to HIV-1 infection and expression of HERV-K proteins generates T-cell cytotoxic responses in many cancers. HERV-K RNA and protein abundance was measured in HIV-1-infected and control cells. In vitro exposure of HIV-1 laboratory-adapted and primary isolates on U87MG cells increased the expression of HERV-K RNA in a dose-dependent manner. HERV-K RNA and protein burdens were significantly increased in HIV-1-producing $\mathrm{H} 9$ cell lines compared to $\mathrm{H} 9$ cells. The expression of HERV-K was synergistically increased in HIV-1-infected PBMCs after stimulation with PMA/ionomycin. Furthermore, the expression of HERV-K in PBMCs, and particularly in $\mathrm{CD4}^{+} \mathrm{T}$ cells, was higher in HIV-1 patients compared to control subjects. The expression of HERV-K might be related to HIV-1 pathogenesis and AIDS-associated cancers.
\end{abstract}

\section{INTRODUCTION}

A LL HUMAN BEINGS CARRY HUMAN ENDOGENOUS RETROVIRUSES (HERVs) inserted in the cellular genome. These sequences comprise up to $8 \%$ of the human genome and are inherited in a parent-to-offspring manner. ${ }^{1-3}$ The Human Genome project revealed that HERV-K (HML-2), the biologically most active endogenous retroviral form, consists of 126 members per haploid genome. ${ }^{4,5}$ The genomes of almost all HERV-K members are rendered defective by the presence of mutations that impair the ability to form infectious viral particles. A recent study showed that HERV-K has increased in copy number by reinfection and thus a pool of replication-competent HERV-K viruses exists in humans. ${ }^{6}$ Recent evidence shows diversified HERV-K RNA sequences in plasma, with a fraction that does not resemble any known HERV-K proviruses and, thus, is likely to be a part of those unfixed HERV-K. ${ }^{7}$ Dewannieux and collaborators proved the infectious capacity of certain HERV-K envelops, such as the almost intact $\mathrm{K} 108 .^{8}$ A new provirus, HERV-K 113, possesses intact open reading frames for all viral genes, and, consequently, is a potential candidate for the production of functional, infectious virions. ${ }^{9}$ HERV-K 113 is polymorphic and exists in about one-third of the human population in Africa and $\sim 5 \%$ in Europe. ${ }^{10,11}$ Activation of replication-competent HERV-K members may cause disease.

Abnormal expression of HERV-K proteins and mobilization of HERV-K elements by retrotransposition may also contrib- ute to the development of pathological conditions. The relation of HERV-K to a pathological condition could be associated with irregular expression of RNA transcripts, viral proteins, viral particles, or the production of autoantibodies to HERV-K components. The Np9 protein coded by type-1 HERV-K (HML-2) proviruses affects tumorigenesis by interacting through the LNX/Numb/Notch pathway. ${ }^{12}$ Activation of HERV-K viruses occurs in several cancer cell lines and tumors. T cell cytotoxic responses to HERV-K antigens have been detected in malignancies such as melanoma and seminoma. ${ }^{13,14}$ Anti-HERV-K antibodies have been detected in $70 \%$ of HIV-1 individuals. ${ }^{15}$ Moreover, an augmented expression of HERV-K transcripts has been observed in patients diagnosed with HIV-1 encephalopathy. ${ }^{16}$ HERV-K RNA genomes, likely contained within viral particles, have been detected in the plasma of HIV-1-infected individuals but rarely detected in hepatatis $\mathrm{C}$ virus $(\mathrm{HCV})$ or normal blood donors. ${ }^{7}$

It is unknown whether the increased expression of HERV$\mathrm{K}$ in HIV-1 requires specific pathological disturbances associated with HIV-1 infection, such as immunosuppression, or if it is directly influenced by infectious HIV-1 particles or viral subproducts. Likewise, the cellular reservoirs where HERV-K becomes activated remain uncertain. The present study tested whether HIV-1 viral particles may induce the expression of HERV-K in CD4-positive HIV-1 permissive, HIV-1 nonpermissive cells and peripheral blood mononuclear cells (PBMCs) in vitro.

AIDS Research Program, Ponce School of Medicine, Ponce, Puerto Rico 00716. 


\section{MATERIALS AND METHODS}

\section{Cell lines and viruses}

Human astrogliomal U87MG cells were maintained in Eagle's minimal essential medium (MEM) (Sigma, St. Louis, MO). The T-lymphoblastoid H9, CEM-NKR cells and the HIV1-producing $\mathrm{H} 9 / \mathrm{HTLV}-\mathrm{III}_{\mathrm{B}}$ and $\mathrm{H} 9 / \mathrm{HTLV}-\mathrm{III}_{\mathrm{RF}}$ cell lines were propagated in RPMI 1640 (Sigma, St. Louis, MO). Media were adjusted to $\mathrm{pH} 7.4$ and supplemented with $2 \mathrm{mM}$ L-glutamine, $10 \%$ fetal bovine serum (FBS), and $1 \%$ antibiotic solution (Sigma, St. Louis, MO). HIV-1 isolates MN and 92BR014 were propagated in fresh PBMCs cultures as described elsewhere. HIV-1 viral titers were determined by real time reverse transcriptase polymerase chain reaction (RT-PCR).

\section{Blood samples}

Sixteen anonymous HIV-1-seropositive blood samples and six seronegative controls were screened for HERV-K expression. Blood samples were provided by the AIDS Research program at Ponce School of Medicine. The HIV-1 viral load in plasma was determined using the Amplicor ultrasensitive test (Roche Diagnostics Corp., Indianapolis, IN). Counts for CD4 and CD8 lymphocytes were measured using a Beckman Coulter cytometer (Becton, Dickinson and Company, Franklin Lakes, NJ) as described elsewhere.

\section{Infectivity studies}

Cell lines and PBMCs were exposed to different titers of $\mathrm{HIV}-1$ isolates HTLV-III $\mathrm{MN}$ or $92 \mathrm{BR} 014$ for $24 \mathrm{~h}$ at $37^{\circ} \mathrm{C}$. When required, PBMCs were stimulated with PMA/ionomycin $24 \mathrm{~h}$ prior to infection. After HIV-1 exposure, supernatants were removed and the cells were repeatedly washed in phosphatebuffered saline (PBS) and grown in supplemented medium for an additional $24 \mathrm{~h}$.

\section{RNA extractions}

After infections, cells were washed three times with PBS and detached with Trypsin-EDTA (Sigma, St. Louis, MO) when required. RNA isolation was performed using the RNeasy blood mini kit (Qiagen). RNA was treated with RNase free DNase (Qiagen, Valencia, CA) for $15 \mathrm{~min}$ at room temperature (RT) and the absence of DNA was confirmed by PCR using the conditions and primers described below. RNA concentration and purity were measured spectrophotometrically at $260 \mathrm{~nm}$ and $280 \mathrm{~nm}$. Viral RNA was extracted from cell-free supernatants using the QIAamp viral RNA mini kit (Qiagen, Valencia, CA) following the manufacturer's procedure.

\section{Real-time RT-PCR}

The primers HERV-Kpol F 5'-TCC CCT TGG AAT ACT CCT GTT TTY GT-3' and HERV-Kpol R 5'-CAT TCC TTG TGG TAA AAC TTT CCA YTG-3' amplify a 297 product corresponding to a conserved reverse transcriptase (RT) region of the HERV-K family. ${ }^{16,17}$ Sequencing analyses of the RT-PCR amplification products in HIV-1-infected plasma samples showed the activation of HERV-K (HML2) and
(HML-3). ${ }^{7}$ The differences in HERV-K mRNA expression were assessed by real time PCR using the QuantiTect Sybr Green RT-PCR kits (Qiagen, Valencia, CA). Then $500 \mathrm{ng}$ of cellular RNA and $0.2 \mu \mathrm{M}$ of each primer were used in a final $25 \mu \mathrm{l}$ reaction. RNA was reverse transcribed for $20 \mathrm{~min}$ at $50^{\circ} \mathrm{C}$. Real time PCR reactions consisted of 50 cycles: $94^{\circ} \mathrm{C}$ for $20 \mathrm{sec}, 50^{\circ} \mathrm{C}$ for $1 \mathrm{~min}, 72^{\circ} \mathrm{C}$ for $30 \mathrm{sec}$, and a fluorescent collection step of $80^{\circ} \mathrm{C}$ for $5 \mathrm{sec}$. Real time analysis was performed with the fluorescent captured at $80^{\circ} \mathrm{C}$ because signal generated by nonspecific PCR products (for example, primers dimers) was determined to be absent. Data were collected and recorded by iCycler iQ software (BioRad, Hercules, CA) and expressed as a function of threshold cycle $\left(C_{\mathrm{t}}\right)$.

Competitor RNA was synthesized in vitro from an HERV$\mathrm{K}$ PCR product inserted in a pcR2.1 clone using the T7 RNA polymerase and the competitor construction kit (Ambion, Austin, TX). Dilutions of competitor RNA $\left(10^{7}\right.$ to $10^{1}$ copies $\left./ \mu \mathrm{l}\right)$ were used as calibrators for real time PCR. RNA relative copy number was extrapolated from standard curves $\left(C_{\mathrm{t}}\right.$ vs. $\log$ starting concentration). The $r^{2}$ was greater than 0.95 for all standard curves. The relative RNA copy number was normalized to the relative abundance of the housekeeping gene pseudo $\beta$-actin 8 (ACTBP8) using the forward primer 5'-CGC CCA GCA CGA TGA AA-3' and reverse primer 5'-CCT CCT ATC CAC ACA GA-3' that span an mRNA segment with no intervening sequences.

\section{Flow cytometry}

Cell cultures were pelleted and washed five times in PBS. Cells were fixed by resuspension in $4 \%$ paraformaldehyde and permeabilized in $1 \%$ Triton $\mathrm{X}-100$ for $30 \mathrm{~min}$. Cells were blocked in permeabilization solution containing $1 \%$ bovine serum albumin for one additional hour. Cells were incubated with the HTDV/HERV-K/Gag-specific monoclonal antibody HERMA-4 for $1 \mathrm{~h}$ at RT. After several washes, cells were incubated with goat antimouse secondary antibody labeled with Alexa Fluor 647 (Invitrogen, Carlsbad, CA) for $1 \mathrm{~h}$ in the dark. For cell surface markers, cells were incubated with FITC-anti CD4, PE-anti-CD8, and PerCP-anti-CD3 (Becton, Dickinson and Company, Franklin Lakes, NJ) for $20 \mathrm{~min}$ in the dark after several washes to remove the secondary antibody. Cells were extensively washed and fluorescent signals were captured by flow cytometry using a FACSCalibur (Becton, Dickinson and Company, Franklin Lakes, NJ). Data was analyzed using the Cell Quest software. The geometric mean fluorescent intensity was used as a measurement of HERV$\mathrm{K}$ Gag protein expression.

\section{Statistical analyses}

The mean number of HERV-K mRNA copy number between HIV-1 infected and mock cells was compared using an independent Student's $t$ test for samples exhibiting normally distributed values. Two-tailed $p$ values were considered significant at $p<0.05$. Correlations between HIV-1 viral load and geometric mean HERV-K Gag fluorescent intensity were analyzed using the Pearson's correlation coefficient in the SPSS software 12.0.1. 


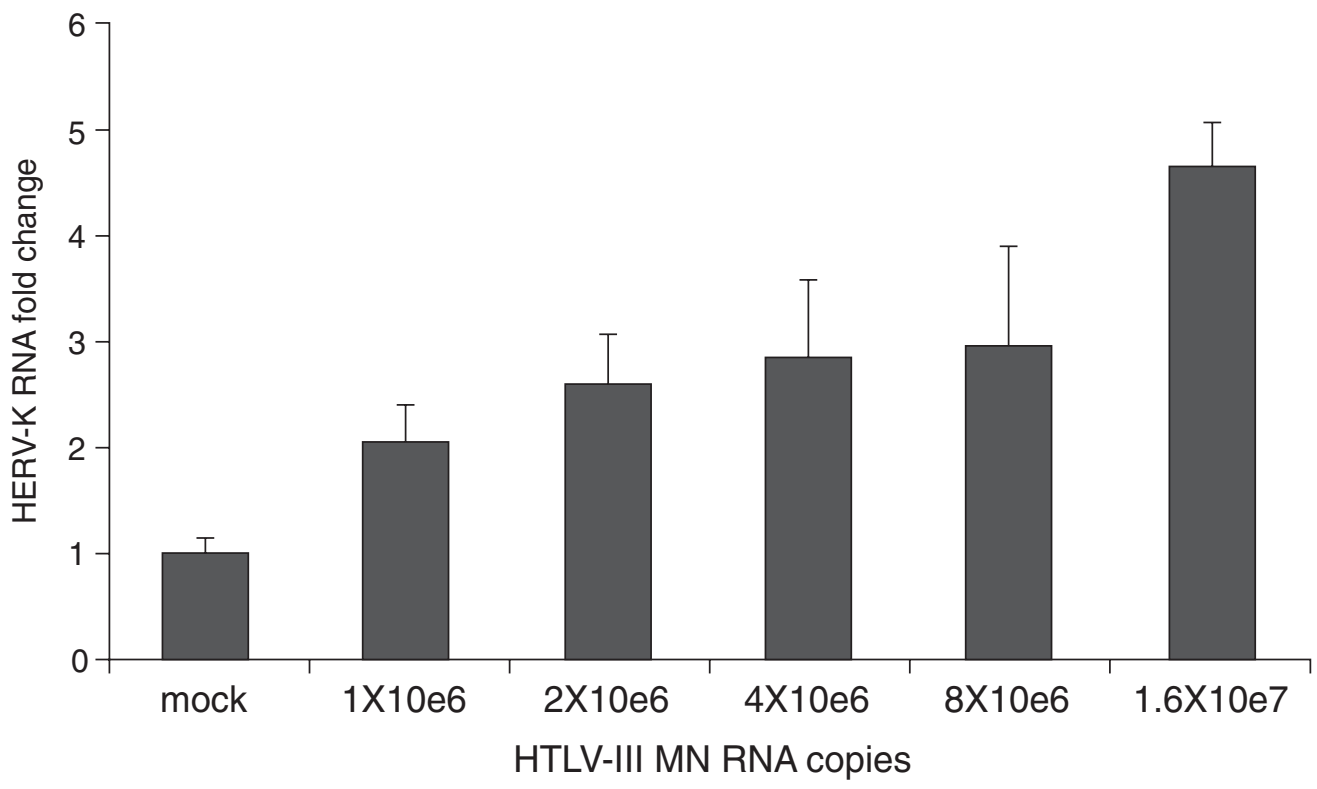

FIG. 1. Dose-dependent activation of HERV-K by HIV-1 viral particles in U87MG cells in vitro. U87MG cells were exposed to increasing doses of HTLV-III MN particles $\left(1 \times 10^{6}\right.$ to $1 \times 10^{7}$ RNA copies $)$ for $24 \mathrm{~h}$. HERV-K cellular RNA was quantified by real time RT-PCR. RNA amounts of the housekeeping gene pseudo- $\beta$-actin did not vary after infections. Data are represented by mean $\pm \mathrm{SD}$ of three separate experiments.

\section{RESULTS}

HIV-1 particles increase cellular HERV-K RNA in a dose-dependent manner in vitro

CD4-positive U87MG cells were exposed to serial dilutions of HIV-1 isolates HTLV-III $\mathrm{MN}_{\text {and }}$ 92BR014 for $24 \mathrm{~h}$. Cellular HERV-K RNA was quantified by real time RT-PCR. Shown are results of the relative expression of HERV-K RNA in U87MG cells exposed to increasung of HIV-1 viral particles (Fig. 1). Upregulation of HERV-K in HIV-1-exposed cells was statistically significant compared to mock-treated cells $(p<$ 0.05). The activation of HERV-K expression was observed to occur in a dose-dependent manner. Similar results were ob- tained for infections of U87MG cells with the primary isolate 92BR014 (data not shown), which may indicate that this effect is not HIV-1 strain-specific. Overexpression of HERV-K in U87MG is likely to be produced by HIV-1 viral components rather than proliferation of $\mathrm{HIV}-1$, since U87MG cells have been shown to restrict HIV-1 replication. ${ }^{18}$

\section{$H I V-1$ replication in stimulated PBMCs synergistically enhances HERV-K expression}

Five million PBMCs were stimulated with PMA/ionomycin for $24 \mathrm{~h}$. Stimulated cells were infected with $1 \times 10^{7}$ viral copies of HTLV-III $\mathrm{MN}_{\mathrm{N}}$ particles. Activation of PBMCs was confirmed by an increase in the extracellular cytokine levels of in- $\mathbf{a}$

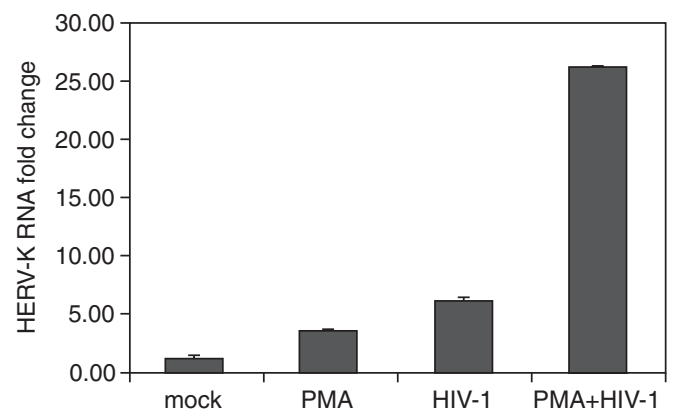

b

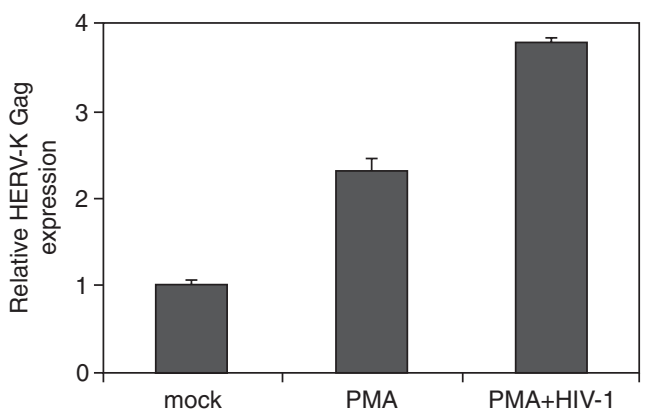

FIG. 2. Upregulation of HERV-K by HIV-1 infection in mock-stimulated or PMA/ionomycin stimulated PBMCs. (a) Relative HERV-K cellular RNA burdens in five million PMA-activated PBMCs infected with HTLV-III MN viral particles $\left(1 \times 10^{7}\right.$ RNA copies) compared to control mock cells. Data are represented by mean and SD of three separate runs. (b) HERV-K Gag protein expression of PMA-stimulated PBMCs after HTLV-III MN infection. Data are represented by the geometric mean fluorescent intensities produced for cellular HERV-K Gag antigens in 10,000 cells. 


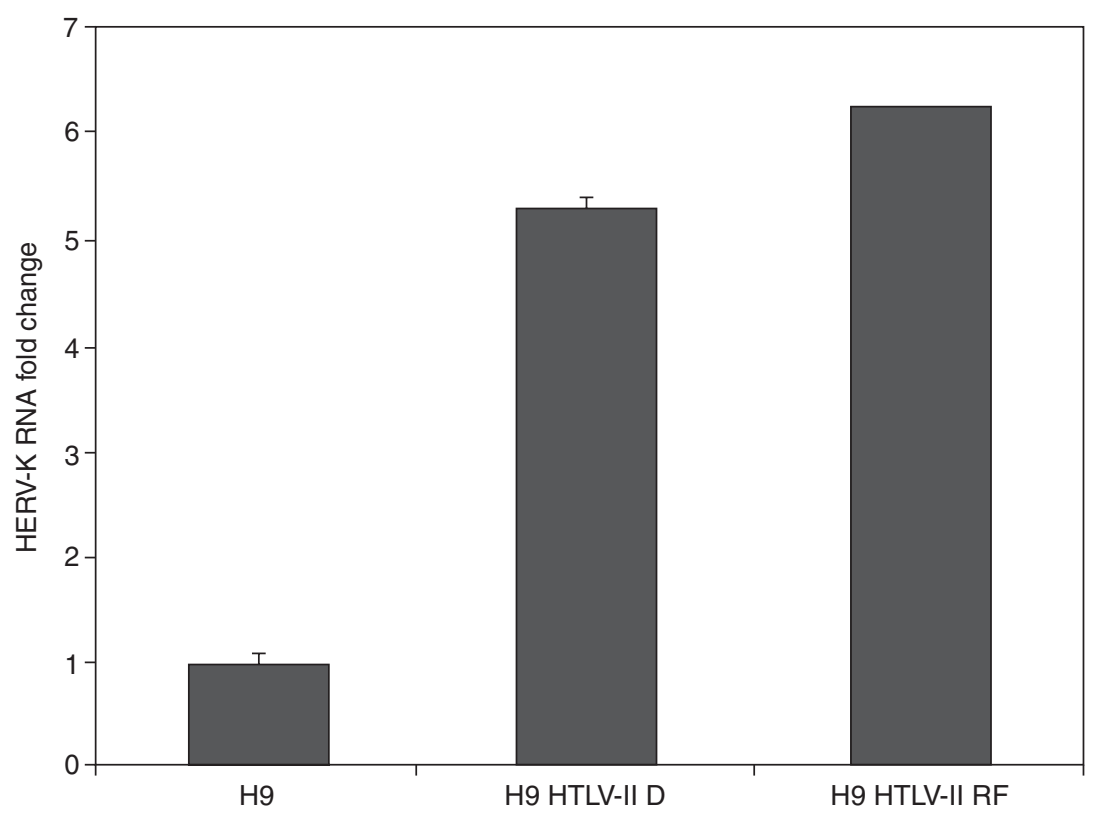

FIG. 3. Expression of HERV-K proteins in chronically infected H9 cell in vitro. Ten millions of H9, H9/HTLV-IIIB, and H9/HTLV-III RF cells were grown in RPMI medium supplemented with 10\% FBS, split 1:2 every 3 days. After 1 week HERV$\mathrm{K}$ protein expression was quantified by flow cytometry. Data are represented geometric mean fluorescent intensity produced by cellular HERV-K Gag proteins in 10,000 cells.

Table 1. HiV-1 Viral Loads and Expression of HerV-K Gag Proteins in HiV-1 Patients and Control Subjects

\begin{tabular}{|c|c|c|c|c|c|c|}
\hline $\begin{array}{l}\text { Patient } \\
\text { ID }\end{array}$ & $\begin{array}{l}\text { HIV-1 } \\
\text { status }\end{array}$ & $\begin{array}{c}\log _{10} H I V-1 \\
R N A \text { load }\end{array}$ & $\begin{array}{c}H E R V-K^{\mathrm{a}} \\
\text { expression } \\
\text { PBMCs } \\
(G M)\end{array}$ & $\begin{array}{c}H E R V-K \\
\text { expression } \\
\text { CD3 T cells } \\
(G M)\end{array}$ & $\begin{array}{c}\text { HERV-K } \\
\text { expression } \\
\text { CD4 T cells } \\
(G M)\end{array}$ & $\begin{array}{c}\text { HERV-K } \\
\text { expression } \\
\text { CD8 T cells } \\
(G M)\end{array}$ \\
\hline 128134 & + & 3.270679 & 43.19 & $\mathrm{ND}^{\mathrm{b}}$ & ND & ND \\
\hline 128137 & + & 4.297213 & 54.54 & ND & ND & ND \\
\hline 128141 & + & 5.875061 & 75.51 & ND & ND & ND \\
\hline 128145 & + & 4.864962 & 36.33 & ND & ND & ND \\
\hline 128148 & + & 4.30311 & 32.82 & ND & ND & ND \\
\hline 128150 & + & 4.277449 & 56.45 & ND & ND & ND \\
\hline 128375 & + & ND & 63.25 & 203.65 & 150.60 & 98.61 \\
\hline 128376 & + & ND & 51.89 & 191.61 & 129.35 & 76.85 \\
\hline 128378 & + & 2.149219 & 59.21 & 122.63 & 116.83 & 76.45 \\
\hline 128380 & + & 1.462398 & 45.87 & 248.17 & 139.02 & 132.97 \\
\hline 128383 & + & 3.418301 & 48.45 & 190.70 & 155.74 & 55.66 \\
\hline 130082 & + & 2 & 40.47 & ND & ND & 121.41 \\
\hline 130062 & + & 2.361728 & 44.70 & ND & ND & 134.11 \\
\hline 130064 & + & 2 & 13.07 & ND & ND & 39.22 \\
\hline 130070 & + & 1 & 50.87 & ND & ND & 152.62 \\
\hline 130058 & + & 4.412074 & 43.28 & ND & ND & 82.59 \\
\hline 140000 & - & 0 & 28.46 & ND & ND & 43.03 \\
\hline 140001 & - & 0 & 24.18 & 101.42 & 86.47 & 42.72 \\
\hline 140002 & - & 0 & 23.02 & 92.96 & 83.49 & 38.56 \\
\hline 140003 & - & 0 & 25.43 & 98.34 & 86.05 & 49.69 \\
\hline 140004 & - & 0 & 18.19 & 114.24 & 85.23 & 36.61 \\
\hline 140005 & - & 0 & 21.19 & ND & ND & 21.19 \\
\hline
\end{tabular}

${ }^{a} \mathrm{HERV}-\mathrm{K}$ protein expression is denoted by the mean geometric fluorescent intensity measured by flow cytometry as described in Materials and Methods.

${ }^{b} \mathrm{ND}$, not determined. 
terleukin (IL)-2, IL-4, IL-6, IL-10, tumor necrosis factor (TNF)$\alpha$, and interferon (IFN)- $\gamma$ as measured with the Human Th1/Th2 Cytokine kit II (Cytometric Bead Array, Becton, Dickinson and Company, Franklin Lakes, NJ) in a FACSArray (Becton, Dickinson and Company, Franklin Lakes, NJ) and HIV-1 replication was monitored by real time RT-PCR (data not shown). Cellular HERV-K RNA (Fig. 2a) was significantly augmented by both cell stimulation and HIV-1 infection $(p<0.01)$.

A quantitative method was established to quantify the HERV-K protein expression by flow cytometry using antibodies specific for the HERV-K Gag antigen Herma-4 (Fig. 2b). No cross-reactivity was found between Herma-4 and HIV antigens using commercial HIV-1 antigen strips from the HIV$\mathrm{AB}^{\mathrm{TM}}$ HIV-1/HIV-2 (rDNA) EIA kit (Abbott Diagnostics, Barceloneta, PR) (data not shown). A synergistic overexpression of HERV-K RNA was observed in stimulated PBMCs infected with HTLV-III MN (Fig. 2a). Overexpression was also confirmed at the HERV-K protein level (Fig. 2b). These results indicated that HERV-K expression is augmented in stimulated
PBMCs. Increased expression of HERV-K RNA in stimulated cells infected with HIV-1 compared to nonstimulated infected cells may indicate that HIV-1 replication in stimulated PBMCs enhances the HERV-K RNA and protein expression. It is also likely that the synergistic HERV-K upregulation is the result of a viral component induced by the boost in the HIV-1 progeny.

\section{Augmented expression of HERV-K proteins in lymphoblastoid cells stably producing HIV-1 virions}

HIV-1-producing and mock lymphoblastoid H9 cell lines were used to determine the levels of HERV-K expression in a chronic model of HIV-1 infection in vitro. Cells were grown in RPMI medium with $10 \%$ FBS for $48 \mathrm{~h}$. HIV-1 particles in the supernatants were quantified by real time RT-PCR (data not shown). A 5- to 6-fold change in HERV-K protein expression was found $(p<0.01)$ in $\mathrm{H} 9$ cells stably producing HIV-1 virions, specifically H9/HTLV-III ${ }_{B}$ and $\mathrm{H} 9 / \mathrm{HTLV}-\mathrm{III}_{\mathrm{RF}}$, compared to control noninfected $\mathrm{H} 9$ cells (Fig. 3). These results suggest (a)

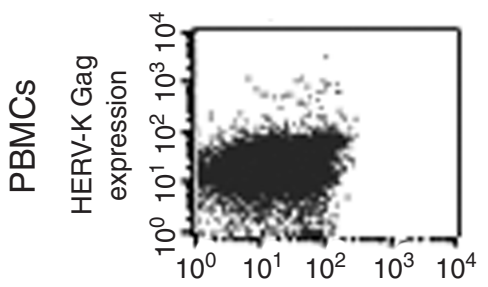

HIV-1 negative
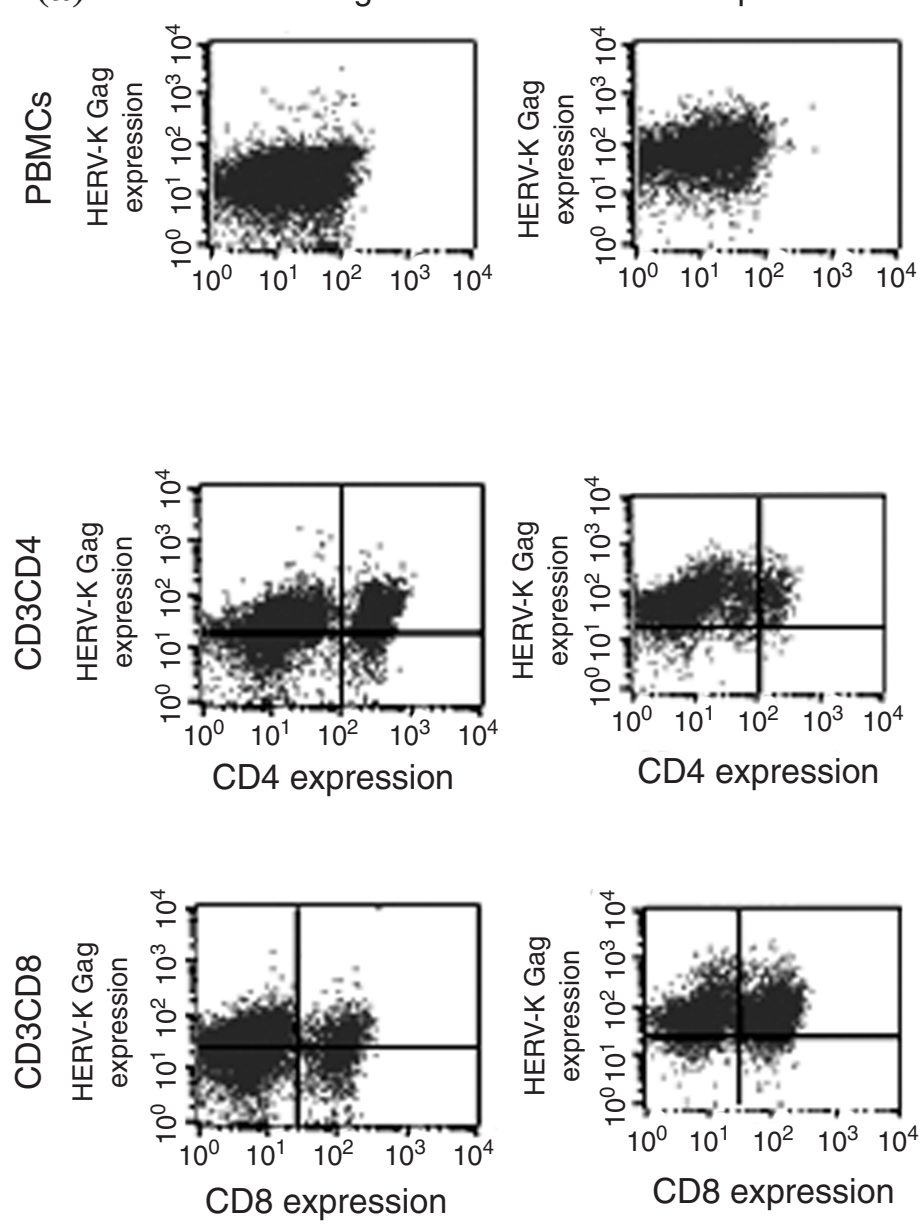

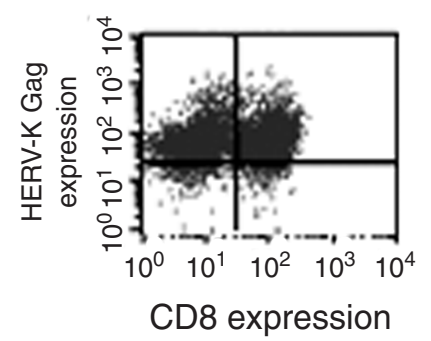

(b)
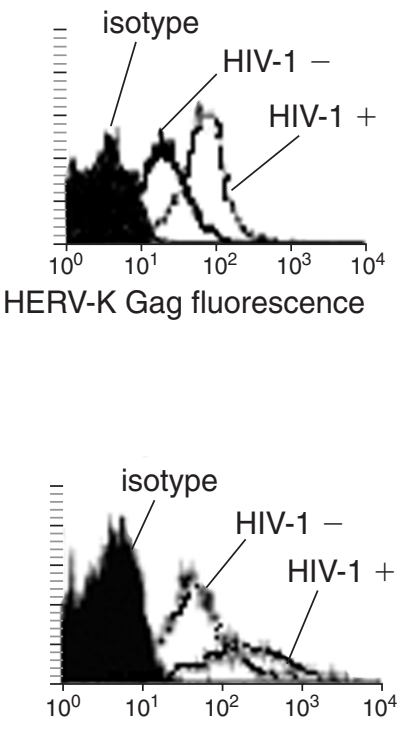

HERV-K Gag fluorescence

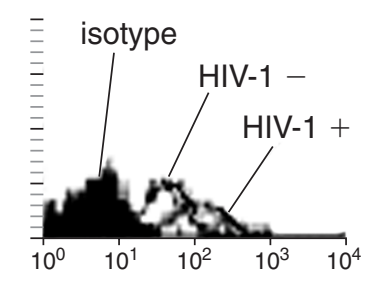

HERV-K Gag fluorescence

FIG. 4. Expression of HERV-K proteins in HIV-1 individuals and seronegative controls. (a) Representative dot blots of the expression of HERV-K proteins ( $y$ axis) in PBMCs from HIV-1-positive patients and control subjects. Expression of HERV-K proteins is also represented in dot blots for CD4 and CD8 lymphocytes. (b) Histograms representing distributions of HERV-K protein expression in general PBMCs and specific CD4 and CD8 lymphocytes from HIV-1-infected individual and seronegative controls. 
that the expression of HERV-K proteins is enhanced in lymphoblastoid cells chronically producing HIV-1 particles. These results suggest that upregulation of HERV-K is likely to occur in both the acute and chronic phases of HIV-1 infection.

\section{Upregulation of HERV-K in PBMCs of HIV-1 patients}

The expression of HERV-K proteins during HIV-1 infection in vivo was further determined by flow cytometry. The mean geometric fluorescent intensity of HERV-K Gag protein expression for each patient is described in Table 1. An increased expression of HERV-K Gag protein was observed in the PBMCs from HIV-1-infected patients compared to seronegative blood donors (Fig. 4). There was an increase in the percentage of PBMCs expressing HERV-K antigens in HIV-1 individuals, and the effect was comparable in the T cell CD4 and CD8 subpopulations (Fig. 4a). Histograms representing fluorescent intensity of the HERV-K Gag protein showed skewedto-right distributions in PBMCs including CD4 and CD8 subsets of HIV-1-infected individuals compared to control subjects (Fig. 4b), indicating upregulation of HERV-K proteins. These data may provide a rationale for the increased detection of antiHERV-K antibodies in HIV-1 individuals. ${ }^{15}$

The expression of HERV-K Gag was correlated with HIV1 viral markers to determine whether HERV-K might play a role during HIV-1 infection. No significant correlation was detected between the HIV-1 titers and HERV-K protein expression in PBMCs of HIV-1 ( $n=14, r=0.32, p=0.25)$. No correlations were found between the HERV-K geometric mean fluorescence and the CD4 or CD8 counts.

\section{DISCUSSION}

HIV-1 viral particles upregulated HERV-K RNA in a dosedependent manner. The HERV-K upregulation was observed in CD4-positive cells, including HIV-1 nonpermissive U87MG cells and permissive $\mathrm{H} 9$ and CD4 T cells in vitro. The HERV$\mathrm{K}$ upregulation was further enhanced in PMA/ionomycin-stimulated PBMCs. This result may indicate that HIV-1 replication in stimulated PBMCs is involved in the activation of HERV$\mathrm{K}$. However, this observation does not explain HERV-K upregulation in HIV-1 nonpermissive U87MG cells. It is likely that increased HIV-1 particle progeny is responsible for the synergy in HERV-K activation observed in stimulated PBMCs. This action is supported by the dose-dependent upregulation of HERV-K in U87MG cells.

An increased expression of HERV-K in HIV-1 patients was generally observed in the PBMCs population, including CD4 and CD8 T cells $(p<0.001)$. The fact that the expression of HERV-K is also enhanced in CD8 T cells may indicate that cells other than CD4-positive cells might be susceptible to the effect of HIV-1 particles, despite a lack of productive viral replication. The possibility that the activation of HERV-K may occur in other cell types cannot be eliminated. On the contrary, the augmented expression of HERV-K transcripts in brain tissue of HIV encephalopathy (HIVE) patients provides additional evidence that other cells may respond to HIV-1 viral components. Other reservoirs for HERV-K activation in HIV-1 infection require further study.
The fact that the patients in this study have been infected for many years may explain the absence of a correlation between HERV-K protein expression and HIV-1 titers, since any cumulative effect of HIV-1 virions among the subjects is almost impossible to determine, even if the viral titers are known at a particular time point. We acknowledge the fact that the measurement of HERV-K expression is purely descriptive and a more accurate quantitation method would be more appropriate for broader and deeper analyses. The lack of association between HERV-K expression and HIV-1 titers may not exclude the possibility that HERV-K affects HIV-1 pathogenesis. However, a direct effect of HERV-K in HIV-1 infection has yet to be discovered. Abnormal expression of HERV-K proteins has been observed in certain cancers, autoimmune diseases, and neurodegenerative disorders, such as HIV encephalopathy (HIVE). ${ }^{11,15,16,19-22}$ Expression of the Np9 protein has been linked to tumorigenesis because it is expressed in mammary carcinoma biopsies, germ cell tumor biopsies, and leukemia blood lymphocytes, but not in normal, nontransformed cells. ${ }^{12}$ Np9 is produced by type-1 HERV-K (HML-2) members that have been previously found in plasma of HIV-1 patients. ${ }^{7}$ Increased expression of HERV-K proteins could be related to AIDS-associated cancer. HERV-K antigens may produce $\mathrm{T}$ cell cytotoxic responses, as observed in certain malignancies. Mechanisms by which HERV-K might also be associated with diseases include the production of somatic mutations resulting in gain or loss of gene function, the transposition of endogenous retroviral promoter regions, which has the potential to influence the expression of linked cellular genes, and the production of viral proteins with biological activities such as the HERV-K18 Env superantigen. ${ }^{24,27}$ Abnormal production of HERV-K proteins may also disrupt immunologic tolerance and may induce autoimmunity.

The activation of putative HERV-K members capable of coding infectious viral particles might accelerate the course of HIV1 progression. Additional studies are necessary to determine the role of HERV-K in HIV-1 infection and the progression to AIDS.

\section{ACKNOWLEDGMENTS}

The HERV-K antibody HERMA-4 was kindly provided by Dr. Klaus Boller, Paul Ehrlich Institut. This work was financially supported by the Research Center for Minority Institutions (RCMI) program (Grant G12RR03050) and the RCMI Publications Office (Grant G12 RR03050-19). The authors acknowledge Bob Ritchie and Michael Khodadoust for reviewing of the English text. The cell lines U87MG, H9, H9/HTLVIIIRF, and H9/HTLV-IIIB and HIV-1 isolates HTLV-III MN and 92BR014 were provided by the NIH AIDS Research and Reference Reagent Program.

\section{REFERENCES}

1. Nelson P, Carnegie P, Martin J, Davari E, Hooley P, Roden D, Rowland-Jones S, Warren P, Astley J, and Murray P: Demystified human endogenous retroviruses. Mol Pathol 2003;56: $11-18$. 
2. Wang-Johanning F, Frost A, Jian B, Epp L, Lu D, and Johanning G: Quantitation of HERV-K env gene expression and splicing in human breast cancer. Oncogene 2003;22:1528-1535.

3. Hughes $\mathrm{J}$ and Coffin J: Human endogenous retrovirus K solo-LTR formation and insertional polymorphisms: Implications for human and viral evolution. Proc Natl Acad Sci USA 2004;101:1688-1672.

4. Barbulescu M, Turner G, Seaman M, Deinard A, Kidd K, and Lenz $\mathrm{J}$ : Many human endogenous retrovirus K (HERV-K) proviruses are unique to humans. Curr Biol 1999;9:861-868.

5. Paces J, Pavlicek A, and Paces V: HERVd: The Human Endogenous Retroviruses Database. Nucleic Acids Res 2002;30:205-206.

6. Belshaw R, Pereira V, Katzourakis A, Talbot G, Paces J, Burt A, and Tristem M: Long-term reinfection of the human genome by endogenous retroviruses. Proc Natl Acad Sci USA 2004;101: 4894-4899.

7. Contreras-Galindo RA, Kaplan MH, Markovitz DM, Lorenzo E, and Yamamura Y: Detection of HERV-K(HML-2) viral RNA in plasma of HIV-1 infected individuals. AIDS Res Hum Retroviruses 2006; 10:979-984.

8. Dewannieux M, Blaise S, and Heidmann T: Identification of a functional envelope protein from the HERV-K family of human endogenous retroviruses. J Virol 2005;79:15573-15577.

9. Turner G, Barbulescu M, Su M, Jensen-Seaman M, Kidd K, and Lenz J: Insertional polymorphisms of full-length endogenous retroviruses in humans. Curr Biol 2001;11:1531-1535.

10. Burmeister T, Ebert A, Pritze W, Loddenkemper C, Schwartz S, and Thiel E: Insertional polymorphisms of endogenous HERVK113 and HERV-K115 retroviruses in breast cancer patients and age-matched controls. AIDS Res Hum Retroviruses 2004;20: $1223-1229$.

11. Moyes D, Martin A, Sawcer S, Temperton N, Worthington J, Griffiths D, and Venables P: The distribution of the endogenous retroviruses HERV-K113 and HERV-K115 in health and disease. Genomics 2005;86:337-341.

12. Armbruester V, Sauter M, Roemer K, Best B, Hahn S, Nty A, Schmid A, Philipp S, Mueller A, and Mueller-Lantzsch N: Np9 protein of human endogenous retrovirus $\mathrm{K}$ interacts with ligand of numb protein X. J Virol 2004;78:10310-10319.

13. Schiavetti F, Thonnard J, Colau D, Boon T, and Coulie P: A human endogenous retroviral sequence encoding an antigen recognized on melanoma by cytolytic $\mathrm{T}$ lymphocytes. Cancer Res 2002;62:5510-5516

14. Rakoff-Nahoum S, Kuebler P, Heymann J, Sheehy M, Ortiz G, Ogg G, Barbour J, Lenz J, Steinfeld A, and Nixon D: Detection of $\mathrm{T}$ lymphocytes specific for human endogenous retrovirus $\mathrm{K}$ (HERV-K) in patients with seminoma. AIDS Res Hum Retroviruses 2006;22:52-56.

15. Löwer R, Löwer J, and Kurth R: The viruses in all of us: Characteristics and biological significance of human endogenous retrovirus sequences. Proc Natl Acad Sci USA 1996;93:5177-5184.

16. Johnston J, Silva C, Holden J, Warren K, Clark A, and Power C: Monocyte activation and differentiation augment human endogenous retrovirus expression: Implications for inflammatory brain diseases. Ann Neurol 2001;50:434-442.
17. Medstrand $\mathrm{P}$ and Blomberg J: Characterization of novel reverse transcriptase encoding human endogenous retroviral sequences similar to type A and type B retroviruses: Differential transcription in normal human tissues. J Virol 1993;67:6778-6787.

18. Gorry PR, Ong C, Thorpe J, Bannwarth S, Thompson KA, Gatignol A, Vesselingh SL, and Purcell DF: Astrocyte infection by HIV1: Mechanisms of restricted virus replication, and role in the pathogenesis of HIV-1-associated dementia. Curr HIV Res 2003;1: 463-473.

19. Karlsson H, Bachmann S, Schroder J, McArthur J, Torrey EF, and Yolken RH: Retroviral RNA identified in the cerebrospinal fluids and brains of individuals with schizophrenia. Proc Natl Acad Sci USA 2001;98:4634-4639.

20. Nakagawa K, Brusic V, McColl G, and Harrison LC: Direct evidence for the expression of multiple endogenous retroviruses in the synovial compartment in rheumatoid arthritis. Arthritis Rheum 1997;40:627-638.

21. Buscher K, Trefzer U, Hofmann M, Sterry W, Kurth R, and Denner JL: Expression of human endogenous retrovirus $\mathrm{K}$ in melanomas and melanoma cell lines. Cancer Res 2005;65:4172-4180.

22. Depil S, Roche C, Dussart P, and Prin L: Expression of a human endogenous retrovirus, HERV-K, in the blood cells of leukemia patients. Leukemia 2002;16:254-259.

23. Galli U, Sauter M, Lecher B, Maurer S, Herbst H, Roemer K, and Mueller-Lantzsch N: Human endogenous retrovirus rec interferes with germ cell development in mice and may cause carcinoma in situ, the predecessor lesion of germ cell tumors. Oncogene 2005; 24:3223-3228.

24. Leib-Mösch C and Seifahrt W: Evolution and biological significance of human retroelements. Virus Genes 1996;11:133-146.

25. Schulte AM, Lai S, Kurtz A, Czubayko F, Riegel AT, and Wellstein A: Human trophoblast and choriocarcinoma expression of the growth factor pleiotrophin attributable to germ-line insertion of an endogenous retrovirus. Proc Natl Acad Sci USA 1996;93: 14759-14764.

26. Sutkowski N, Conrad B, Thorley-Lawrson DA, and Huber BT: Epstein-Barr virus transactivates the human endogenous retrovirus HERV-K18 that encodes a superantigen. Immunity 2001;15: 579-589.

27. Taruscio D and Mantovani A: Human endogenous retroviral sequences: Possible roles in reproductive physiopathology. Biol Reprod 1998;59:713-724.

Address reprint request to:

Rafael Contreras-Galindo

Division of Infectious Diseases

Department of Internal Medicine

University of Michigan

5220 MSRB III

1150 West Medical Center Drive Ann Arbor, Michigan 48109-0640

E-mail: rafaelc@med.umich.edu 
This article has been cited by:

1. Marian P Laderoute, Antonio Giulivi, Louise Larocque, Deana Bellfoy, Yangxun Hou, Hong-Xing Wu, Keith Fowke, Jun Wu, Francisco Diaz-Mitoma. 2007. The replicative activity of human endogenous retrovirus K102 (HERV-K102) with HIV viremia. AIDS 21:18, 2417-2424. [CrossRef]

2. Rafael Contreras-Galindo, Sharilyn Almodóvar-Camacho, Sandra González-Ramírez, Eric Lorenzo , Yasuhiro Yamamura . 2007. Short Communication: Comparative Longitudinal Studies of HERV-K and HIV-1 RNA Titers in HIV-1-Infected Patients Receiving Successful versus Unsuccessful Highly Active Antiretroviral TherapyShort Communication: Comparative Longitudinal Studies of HERV-K and HIV-1 RNA Titers in HIV-1-Infected Patients Receiving Successful versus Unsuccessful Highly Active Antiretroviral Therapy. AIDS Research and Human Retroviruses 23:9, 1083-1086. [Abstract] [PDF] [PDF Plus] 